\title{
Consideration of creep of geosynthetic materials in the numerical modeling
}

\author{
Evgeniy Fedorenko ${ }^{1, *}$ \\ ${ }^{1}$ MIAKOM Ltd., Popov str., 4, Saint Petersburg, 197341, Russia
}

\begin{abstract}
There is the opportunity to specify the nonlinear behavior of «Geogrid» element that is intended for modeling of geosynthetic materials and the opportunity to take into account creep in the latest version (2017) of the Plaxis program. The numerical modeling gives the possibility to make a calculation taking into account not only geosynthetic materials creep, but also soils creep. The rheological behavior of «Geogrid» element is based on the theory of the idealized creep of geosynthetic materials and the theory of Kelvin-Voigt. It is necessary for calculations to set the stiffness under short term tension and stiffness along the isochrone of estimated time and a specific parameter retardation time. An example of the calculation of an embankment on soft soils is presented in the report with respect to soils strengthening in the consolidation process, soils creep (model SoftSoil) and creep of geogrids. The required strength of a geosynthetic layer is determined in dependence on these factors.
\end{abstract}

\section{Linear-dependent behavior without limiting the tensile strength value}

This option requires the value of axial stiffness EA introduction. In this case, the behavior of the reinforcing layer is described by the graph in Fig. 1 .

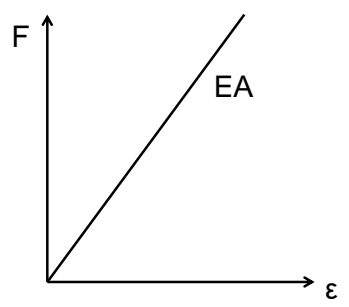

Fig. 1. Elastic behavior.

The peculiarity of the behavior in this mode of setting parameters is that during the safety calculation there is a gradual increase in deformations of geogrids until the loss of convergence of the solution and stop of the calculation phase. Of course, the deformations and stresses obtained at the Safety stage are unrealistic and are not used to analyze the results; also, the values of the Safety factor in this case will be ambiguous.

\footnotetext{
*Corresponding author: evgeniy@miakom.ru
} 
The strength reduction method (SRM) has its own characteristics [1] and can not be considered as a reference in assessing stability, because it depends heavily on many parameters (initial state, soil model, the correctness of the choice of strength parameters and the type of behavior, etc.). It is necessary to perform mutual verification of the results using the method of strength reduction (numerical calculation) by conventional methods of limiting equilibrium (analytical calculation) [2] in stability calculations.

However, as stated above, the analytical method of calculation does not take into account the stiffness parameters, and is based only on the value of the limiting computational effort F. Thus, for the correct comparison of the numerical and analytical calculations, it is necessary to enter the limit force value $\mathrm{F}$ into the calculation, i.e. to use the behavior of the geogrid element Elastoplastic.

\section{Linearly-dependent behavior with the limit of ultimate strength values}

The difference between this type of behavior and the previous one is the input of the second parameter - the limit value of the design load $\mathrm{N}$.

Then the behavior of the geogrid element will be described by the graph in Fig. 2.

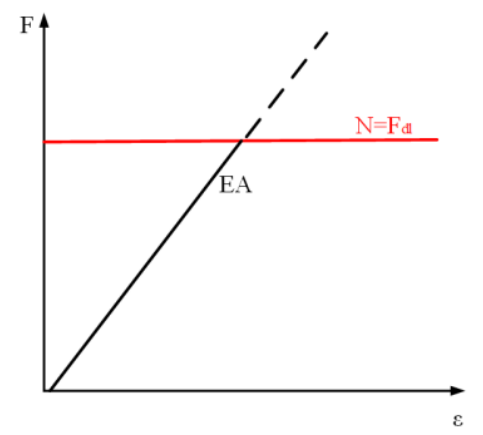

Fig. 2. Elastoplastic behavior.

The program Plaxis, in excess of the specified limit value of strength $\mathrm{N}$ stops the calculation, or it is seen that movements are growing and the rate of $\Sigma \mathrm{M}_{\text {stage }}$ is not changing. However, in some cases, it may be that the calculation was made with the excess of the specified strength of the reinforcing layer. This point should be checked against the tensile force diagram in the geogrid element (Axial forces N). Fig. 3 shows a variant with a nonexceeding value of $\mathrm{N}$, while Fig. 4 shows exceeding the specified strength.

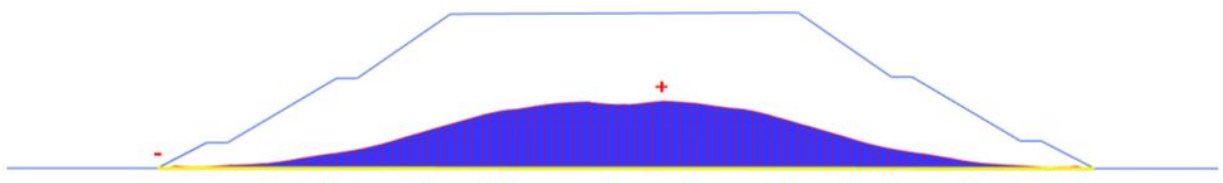

Fig. 3. Tensile force diagram (without exceeding of the specified tensile strength).

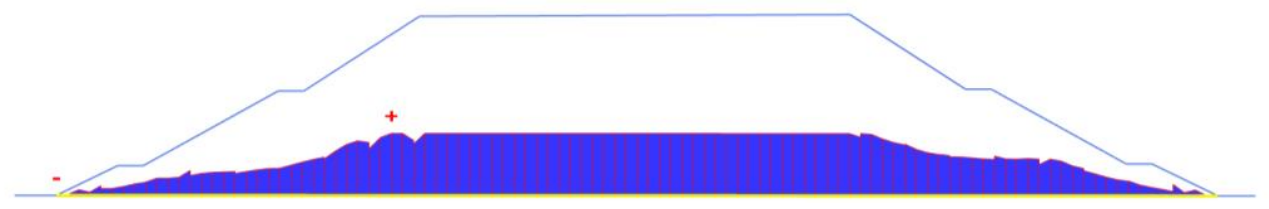

Fig. 4. Tensile force diagram (with exceeding of the specified tensile strength). 


\section{Nonlinear behavior with the limit of ultimate strength values}

The Elastoplastic $(\mathrm{N}-\varepsilon)$ behavior mode enables a user to enter the values of laboratory testing of geosynthetic material for breaking strength and to take into account its nonlinear behavior.

Elongation values assume only the convex shape of the graph, without plots of variable curvature, i.e. only reduction of the ratio of the load increment to the elongation increment is allowed. This requirement is quite reasonable, given that we are talking about polymeric materials and their increase in rigidity in the process of testing depends on the speed of load application and cannot be taken into account in the calculations for reasons of safety [3].

\section{Linear-dependent behavior with limiting of ultimate strength value (creep consideration)}

Such behavior allows estimating both deformations: during construction and during exploitation within the given time during modeling (calculation).

The Kelvin-Voigt model is used to describe the behavior of the reinforcing material in time (Fig. 5).

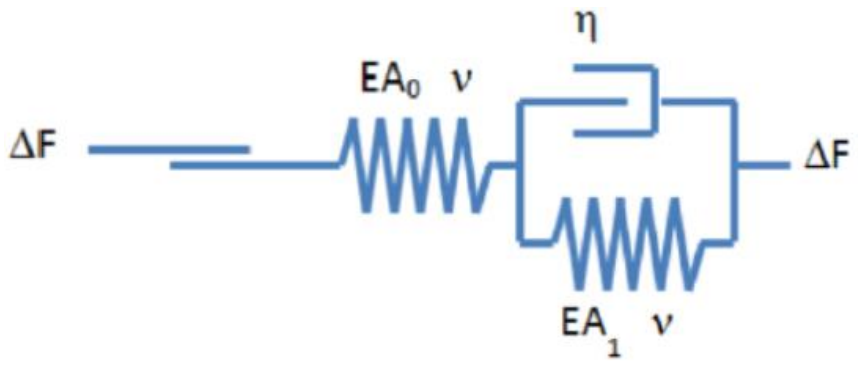

Fig. 5. The Kelvin-Voigt model.

As can be seen from the scheme the model [4] uses such parameters as follows:

-the initial axial stiffness $\mathrm{EA}_{0}=\mathrm{EA}_{\text {sport }}$;

- long - term axial stiffness along isochrone corresponding to the behavior through the required amount of time $\mathrm{EA}_{1}=\mathrm{EA}_{\mathrm{long}}$;

- viscosity $\eta$, i.e. the parameter taking into account the rigidity in time (Retardation time).

The correlation of the parameters can be presented as follows:

$$
\begin{aligned}
t_{\text {ret }} & =\frac{\eta}{E} \\
U_{\text {short }} & =\frac{F}{E A_{\text {short }}} \\
U_{\text {long }} & =\frac{F}{E A_{\text {long }}}
\end{aligned}
$$

Table shows the input window of the parameters of the geogrid element for Visco elastic (time dependent) behavior.

Table 1. Parameters Visco elastic (time dependent) behavior.

\begin{tabular}{|l|l|}
\hline Properties & \\
\hline initial axial stiffness EA $\mathrm{E}_{\text {sport }}$ & $1200 \mathrm{kN} / \mathrm{m}$ \\
\hline long-term axial stiffness $\mathrm{EA}_{\text {long }}$ & $900 \mathrm{kN} / \mathrm{m}$ \\
\hline strength limitation & $50 \mathrm{kN} / \mathrm{m}$ \\
\hline retardation time & 8000 day \\
\hline
\end{tabular}


The reference manual to the program [4] provides an explanatory graph (Fig. ), from which it follows that the axial stiffness of the initial $\mathrm{EA}_{\text {short }}$ corresponding to the isochrone with zero time value or the usual curve of laboratory tests for tensile strength, differs from the axial stiffness of the long-term EA $_{\text {long }}$ corresponding to the isochron with given time (e.g. for 120 years).

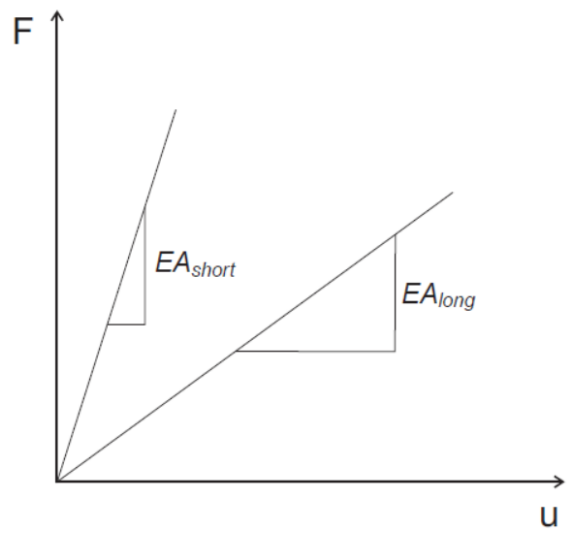

Fig. 6. Graphical interpretation of initial and long-term axial rigidity.

To assign a parameter Retardation time, which is the reference time and is defined as the intersection point of the linear extrapolation of the time dependence of the elongation with the maximum elongation at the estimated time (for example, service lifetime of a construction).

To determine the $\operatorname{Tr}$ (Retardation time) parameter, we can use the following method, according to the isochronic diagrams (Fig. ) to construct dependence diagram of elongation in time (Fig. on the right) and extend the linear dependence of this graph to its intersection with the maximum deformation.

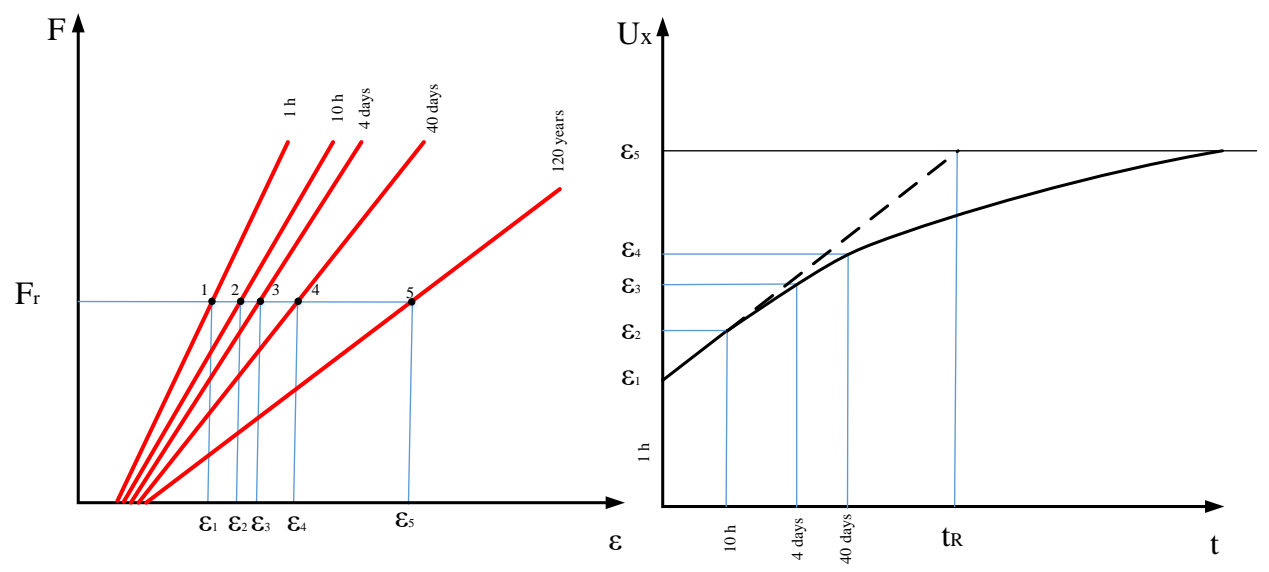

Fig. 7. The key to the definition of the parameter Retardation time.

The geogrid element (Fig. ) was tested by loading it at different sets of parameters (table 2). The results showed a complete coincidence of its behavior with the analytical calculation.

The modeling was performed under a tensile load of $F=10 \mathrm{kN} / \mathrm{m}$ for the following points of time: $0,3,5,10,15,20,25,30,40$ days. In this case, the set reference time for transition from short-time to long-term axial rigidity (Retardation time) for an example was assumed equal to $t_{R}=5$ days. This principle of functional check of an element allows to 
estimate the value of deformation at the initial loading corresponding to the construction period (construction deformation), and deformations that are realized at constant load in time, i.e. associated with creep.

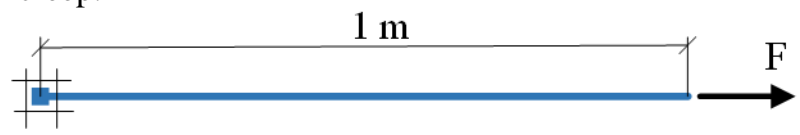

Fig. 8. Design diagram.

Table 2. The set of parameters of the geogrid element.

\begin{tabular}{|c|c|c|}
\hline Parameter & Unit & Value \\
\hline EA $_{\text {short }}$ & $\mathrm{kN} / \mathrm{m}$ & 700 \\
\hline EA $A_{\text {long }}$ & $\mathrm{kN} / \mathrm{m}$ & 700 \\
\hline $\mathrm{N}$ (limit) & $\mathrm{kN} / \mathrm{m}$ & 40 \\
\hline Retardation time $\mathrm{t}_{\mathrm{R}}$ & day & 5 \\
\hline
\end{tabular}

For conditionally instantaneous application of load (simulation of the construction period, when there is a gradual loading of reinforcing elements) on the initial axial stiffness

$$
U_{x}^{\text {initial }}=\frac{F}{E A_{\text {short }}}=\frac{10}{30}=0,333 \mathrm{~m}
$$

Time point 50 days (outside reference time $t_{R}=5$ days) for the static constant load in force during this period $\mathrm{F}=10 \mathrm{kN} / \mathrm{m}$ for long-term axial stiffness $\mathrm{EA}_{\text {long: }}$ :

$$
U_{x}^{\text {long }}=\frac{F}{E A_{\text {long }}}=\frac{10}{10}=1 \mathrm{~m}
$$

Thus, the program allows to determine the value of initial deformation of geosynthetic materials $\mathrm{U}_{\mathrm{x}}{ }^{\text {initial }}=0.333 \mathrm{~m}$ (building deformation) and the value of deformation for this stress level at any time (operational deformations), including the maximum possible deformation $\mathrm{U}_{\mathrm{x}}^{\text {long }}=1 \mathrm{~m}$.

Picture 9 (on the left) presents a graphical interpretation of the initial EA $\mathrm{Ashrt}_{\text {shd a long }}$ axial stiffness $\mathrm{EA}_{\text {long }}$ with the corresponding strain at a given stress level $\mathrm{F}=10 \mathrm{kN} / \mathrm{m}$. The right part of Fig. shows the theoretical curve of deformations in time (creep) and the parameter $t_{R}$ (Retardation time).

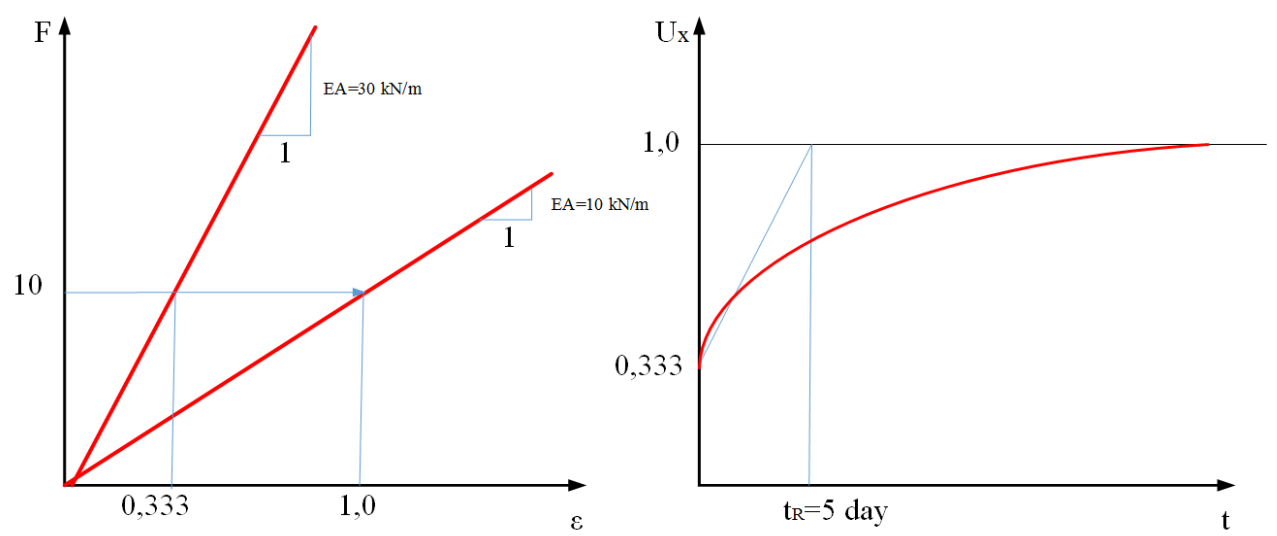

Fig. 9. Graphical representation of the calculation parameters. 
Thus, it can be clearly seen that the geogrid element with the Visco elastic (time dependent) behavior type makes it possible to estimate creep deformations, i.e. elongation in time at a constant stress level.

The graph of the dependence of deformations in time that has been obtained by the results of calculations is now shown in Fig. 0.

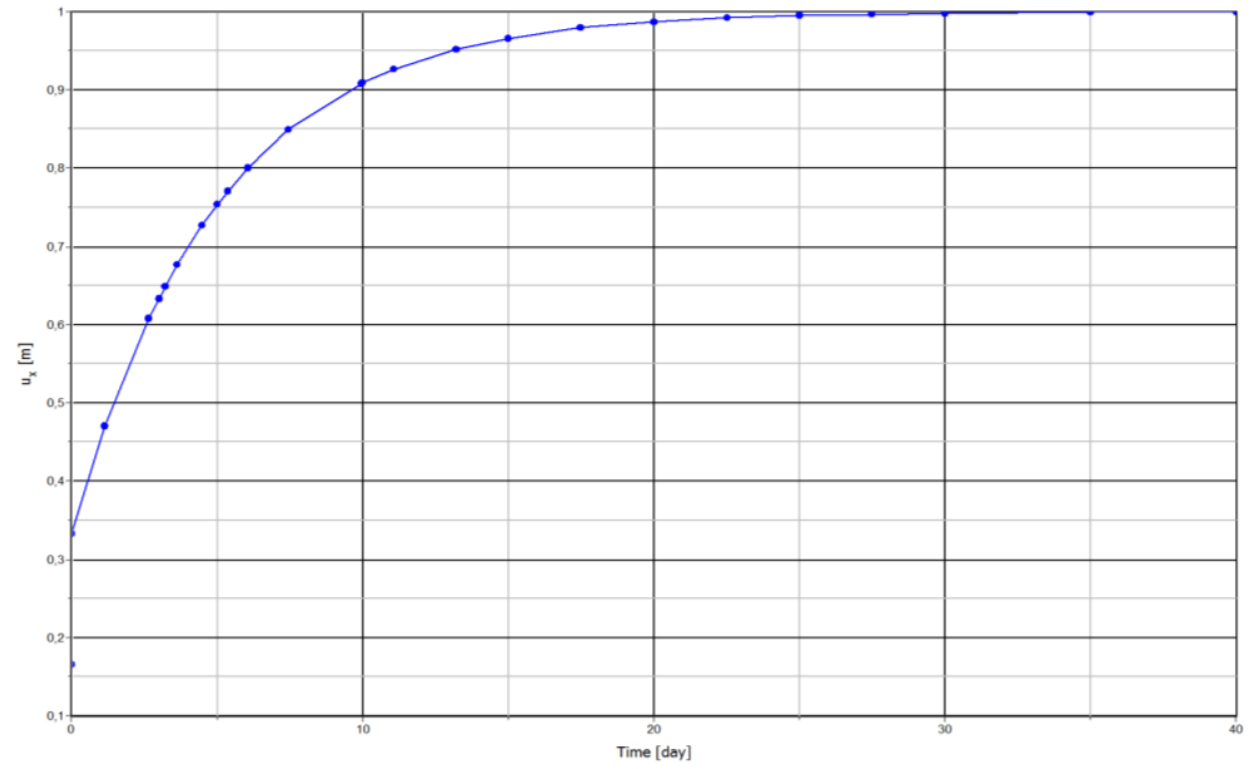

Fig. 10. The results of the modeling of creep deformation.

\section{Conclusion}

Consideration of the creep of geosynthetic materials is particularly important for such structures as reinforced retaining walls [5]. The complexity for analytical calculations is the need to take into account the transition of soils from the state of pressure in the state of rest to the state of active pressure. In addition to the above, it is necessary to take into account the deformation of the reinforcing elements.

Numerical modeling allows solving this difficult problem, but until 2017 there was no possibility to consider creep in this calculation. The set of parameters Visco elastic for the geogrid element in the Plaxis software package allows determining the strain value of the geosynthetic reinforcing layer, and the structure itself, at any time. For the correct input parameters, it is necessary to have isochrone graphics of materials creep testing.

\section{References}

1. E.V. Fedorenko, Transport of the Russian Federation 6(49), 24-26 (2013)

2. E.V. Fedorenko, Geotechnics and geosynthetics in questions and answers (Guide, SPb, 2016)

3. T.-L. Gouw, Importance of elongation factor in determining geosynthetics stiffness for finite element calculation, International Confernce on Landslides and Slope Stability 1

4. Reference Manual PLAXIS 2D (2017)

5. T.S. Vavrynyuk, E.V. Fedorenko, Transport construction 10, 25-28 (2013) 